\title{
Maternal Cardiopulmonary Resuscitation
}

1. Major causes of cardiac arrest during pregnancy: AFE, hemorrhage (DIC, placental abruption, placenta previa, uterine atony), iatrogenic (anesthetic complications, hypermagnesemia, medication errors or allergy), preexisting heart disease (congenital or acquired), pregnanc-induced HTN, sepsis, trauma, PE

2. If prior to delivery, left uterine displacement must be maintained and aortocaval compression avoided during resuscitation

3. ABCs as in non-pregnant (airway, ventilation, circulation)

(a) The 2015 American Heart Association guideline recommends the same hand position for Chest compressions in pregnant women and nonpregnant adults. Manual uterine displacement during CPR to avoid aortocaval compression, optimize venous return, and generate adequate stroke volume

4. Standard cardiopulmonary resuscitation algorithms and pharmacologic therapy without modification

5. If resuscitative efforts are unsuccessful, emergency hysterotomy (perimortem cesarean) must be initiated at four minutes postarrest as it may be impossible to resuscitate the mother until adequate venous return is restored

(a) Decision for delivery depends on gestational age, features of cardiac arrest (e.g. duration of arrest and hypoxemia) and professional setting (skills of surgeon, anesthesia provider, neonatologist, and presence of support personnel)

(i) $<20$ weeks gestation, hysterotomy performed for maternal resuscitation, but fetus not viable

(ii) $>24$ weeks, delivery improves chances of survival for both mother and baby

(iii) Best infant survival when delivery occurs within 5 min of maternal arrest 